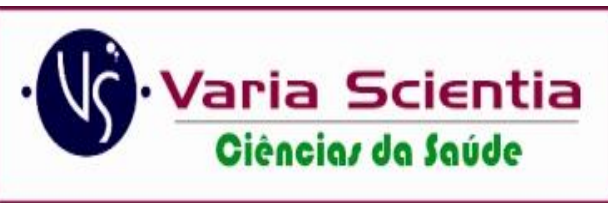

e-ISSN 2446-8118

\title{
ÉTICA EM PESQUISA E RESPONSABILIDADES SOCIAIS DOS PESQUISADORES: UMA PERSPECTIVA DO CEP UNIOESTE
}

\author{
RESEARCH ETHICS AND SOCIAL RESPONSIBILITIES OF RESEARCHERS: A \\ PERSPECTIVE FROM CEP UNIOESTE
}

\section{ÉTICA DE LA INVESTIGACIÓN Y RESPONSABILIDADES SOCIALES DE LOS INVESTIGADORES: UNA PERSPECTIVA DESDE CEP UNIOESTE}

\author{
Dartel Ferrari Lima ${ }^{1}$ \\ Lohran Anguera Lima² \\ Vilmar Malacarne ${ }^{3}$ \\ João Fernando Cristofoletti ${ }^{4}$
}

RESUMO: As questões da ética em pesquisa estão se tornando, de modo global, cada vez mais, importantes no gerenciamento da pesquisa. O Comitê de Ética em Pesquisa com Seres Humanos da Universidade Estadual do Oeste do Paraná (CEP Unioeste), tem regulado no âmbito da Unioeste e da região Oeste do Paraná, a análise ética de propostas de pesquisa que envolvem seres humanos, a fim de potencializar a autonomia do pesquisador em pesquisar e proteger os participantes da pesquisa. O objetivo desse estudo, foi analisar, no quinquênio de 2015 a 2019, os procedimentos decisórios de revisões de protocolos de pesquisa submetidos a inspeção do CEP Unioeste, procurando (re)conhecer as potencialidades nas decisões desse Comitê de ética. As informações

\footnotetext{
${ }^{1}$ Universidade Estadual do Oeste do Paraná. Fisioterapeuta, Mestre em Engenharia Biomédica pela Universidade Tecnológica Federal do Paraná e Doutor em Ciências pela Faculdade de Medicina da Universidade de São Paulo. Especialista (Residência) em Fisioterapia Neurofuncional e Fisioterapia Ortopédica Traumatofuncional (COFFITO). Especialista Lato Sensu em Biologia do Esporte e Preparação Física. Atualmente é professor Adjunto na Universidade Estadual do Oeste do Paraná, Brasil.

2 Santa Casa de Misericórdia de São Paulo. Departamento de Ortopedia e Traumatologia. Médico formado pela Universidade Estadual do Oeste do Paraná. Ortopedista e traumatologista pela Santa Casa de São Paulo (Pavilhão Fernandinho Simonsen). Integrou a equipe médica do campeonato mundial de surfe (WSL) no Rio de Janeiro (2012 e 2013). Membro da Sociedade Brasileira de Ortopedia e Traumatologia (SBOT), da North American Spine Society (NASS) e da AOSpine.

${ }^{3}$ Universidade Estadual do Oeste do Paraná. Graduado em Filosofia Licenciatura Plena, mestre em Educação e doutor em Educação pela Universidade de São Paulo (2007). Atualmente é professor Associado da Universidade Estadual do Oeste do Paraná. Coordenador geral do Núcleo de Pesquisa em Ensino de Ciências e Matemática - NUPECIM.

${ }^{4}$ Universidade Estadual do Oeste do Paraná. Graduado em Educação Física (Bacharelado e Licenciatura) e em Filosofia (Bacharelado) pela Universidade Estadual do Oeste do Paraná (2012), mestrado em Educação Física e doutor em Educação pela University of London (2010). Atualmente é professor adjunto D na Universidade Estadual do Oeste do Paraná, atuando nos curso de bacharelado e licenciatura Educação Física e no programa de pós-graduação em Educação em Ciências e Educação Matemática. Coordenou o Comitê de Ética em Pesquisa com Seres Humanos da Unioeste entre 2012 e 2016. É vice-líder do grupo de pesquisa História Intelectual e Historiografia desde 2010 e, desde 2018, também participa do grupo de pesquisa Corpo, Cultura e Atividade física. Tem experiência na área de Filosofia, com ênfase em Epistemologia e Filosofia da Ciência, e na área de Educação Física, com ênfase em Sociologia do Esporte e História do Corpo.
} 
para as análises foram obtidas do Relatório de Desempenho do CEP Unioeste, disponibilizadas na Plataforma Brasil, uma base nacional e unificada de registros de pesquisas envolvendo seres humanos, coordenada pela Comissão Nacional de Ética em Pesquisa (Conep).

Os procedimentos adotados pelo CEP Unioeste, mostraram redução de pendências procedimentais na primeira fase das análises, celeridade do processo de tramitação e ampliação anual do número de processos analisados.

DESCRITORES: Ética em pesquisa; Análise de vulnerabilidade; Humanos.

ABSTRACT: Global research ethics issues are becoming increasingly important in research management. The Human Research Ethics Committee of the State University of Western Paraná (CEP Unioeste), has regulated within the scope of Unioeste and the Western region of Paraná, the ethical analysis of research proposals involving human beings, in order to enhance the researcher's autonomy in research and the protection of research participants. In this work, a procedural analysis of the decision-making reviews of CEP Unioeste was carried out, in the five-year period from 2015 to 2019, seeking to (re) discover the potential of this ethics committee. The information for the analyzes was obtained in the CEP Unioeste Performance Report, made available (restricted use) on Plataforma Brasil, a national and unified base of research records involving human beings, coordinated by the National Research Ethics Commission (Conep). The procedures adopted by CEP Unioeste, showed a reduction in procedural pending issues in the first phase of the analyzes, speed of the processing process and expansion of the number of analyzed cases.

DESCRIPTORS: Ethics research; Vulnerability analysis; Humans.

RESUMEN: Los problemas globales de ética de la investigación son cada vez más importantes en la gestión de la investigación. El Comité de Ética en Investigación Humana de la Universidad Estatal de Paraná Occidental (CEP Unioeste), ha regulado dentro del alcance de Unioeste y la región occidental de Paraná, el análisis ético de las propuestas de investigación que involucran a seres humanos, con el fin de mejorar La autonomía del investigador en la investigación y la protección de los participantes en la investigación. En este trabajo, se llevó a cabo un análisis de procedimiento de las revisiones de toma de decisiones de CEP Unioeste, en el período de cinco años de 2015 a 2019, buscando (re) descubrir el potencial de este comité de ética. La información para los análisis se obtuvo en el Informe de Desempeño del CEP Unioeste, puesto a disposición (uso restringido) en Plataforma Brasil, una base nacional y unificada de registros de investigación que involucra seres humanos, coordinada por la Comisión Nacional de Ética de Investigación (Conep). Los procedimientos adoptados por CEP Unioeste, mostraron una reducción en los problemas procesales pendientes en la primera fase de los análisis, la velocidad del proceso de procesamiento y la expansión del número de casos analizados.

DESCRIPTORES: Ética en investigación; Análisis de vulnerabilidad; Humanos.

\section{INTRODUÇÃO}

Ao longo do tempo, a ética na pesquisa se debruça na tentativa de equilibrar a tensão existente entre a liberdade acadêmica para pesquisar e a responsabilidade do pesquisador com os meios adotados para atingir os resultados, ou seja, a mediação entre o avanço do conhecimento e o respeito aos participantes da pesquisa, ainda se depara com alguns acontecimentos controversos.
Entre outras tantas situações, a visão geral das questões contemporâneas da ética em pesquisa destaca representações socioculturais da ética com base em uma construção burocrática e legal, centralizada na discussão acadêmica e pública, que exige a responsabilidade e a reflexão dos atores envolvidos para consolidar um modelo consistente com as responsabilidades éticas na pesquisa. Nesse sentido, o ato de pesquisar ainda causa turbulências nas condutas de 
proteção ao participante da pesquisa e na garantia dos direitos dos participantes.

A Universidade Estadual do Oeste do Paraná, criada em 1987, constitui-se uma Instituição Pública de Ensino Superior de formato multicampi, abrangendo as regiões Oeste e Sudoeste do Paraná, com Reitoria centralizada na cidade de Cascavel. Nascida da junção de quatro faculdades municipais da região Oeste paranaense, Cascavel, Toledo, Foz do Iguaçu e Marechal Cândido Rondon, no ano de 1999, incorporou o Campus de Francisco Beltrão. A instituição atende a um conjunto populacional regional maior de um milhão de habitantes. A produção de pesquisa da Unioeste é maior produção da região Oeste do Paraná e, uma das maiores do Estado.

No ano de 2000, criou o CEP Unioeste, composto por 18 docentes da Unioeste, enraizados em todas as áreas do conhecimento humano, representando os cinco campi da Universidade. Outros três membros leigos, representam os participantes da pesquisa, constituindo o Controle Social do sistema CEP/Conep. O CEP Unioeste, atende todos os campi da Unioeste, o Hospital Universitário do Oeste do Paraná (HUOP) e outras Instituições de Pesquisa conveniadas; é uma instância colegiada multidisciplinar e autônoma, de natureza consultiva, deliberativa e educativa, com função de defender os interesses dos participantes envolvidos em pesquisa e contribuir para o desenvolvimento de pesquisas de acordo com os padrões éticos estabelecidos pela regulação Federal.

Aqui, nosso propósito, foi analisar, no quinquênio de 2015 a 2019, os procedimentos decisórios de revisões de protocolos de pesquisa submetidos a inspeção do CEP Unioeste, procurando (re)conhecer as potencialidades nas decisões desse Comitê de ética. As informações para as análises foram obtidas do Relatório de Desempenho do CEP Unioeste, disponibilizadas na Plataforma Brasil, uma base nacional e unificada de registros de pesquisas envolvendo seres humanos, coordenada pela Comissão Nacional de Ética em Pesquisa (Conep).

\section{Desafios e dificuldades na análise dos protocolos de pesquisa}

As questões éticas em pesquisas são o foco deste ponto de vista que se concentra em argumentar aspectos de interação do pesquisador com a pesquisa, mediado pelos comitês de ética ${ }^{1}$. Mais especificamente, se preocupa com o desempenho do papel decisório nas questões éticas, enfrentados por pesquisadores e a sociedade e influenciados pelas pressões econômicas, sociais e culturais exercidas sobre aqueles que pesquisam, muitas vezes, levando a transgressão dos cuidados com a pesquisa ${ }^{2}$.

Atualmente, há uma ênfase acadêmica em ser cientificamente produtivo. A produção, geralmente é pesada, ora pela qualidade, ora pela quantidade. Habitualmente, o apressamento para resultados tende a prejudicar o desempenho de qualidade. Assim, o apressamento tende a aumentar a velocidade da produção em detrimento da qualidade, potencializando faltas danosas na geração de respostas e de produtos $^{3}$.

A definição de metas para pensar, compartilhar e valorizar a integridade da construção do conhecimento não é apenas um esforço pedagógico, mas um exercício de responsabilidade. No campo da ética na pesquisa, é necessário pensar a ética com o desafio de ser capaz de decidir quais valores que se deseja e qual é o preço justo a pagar. Trata-se, portanto, agregar os interesses dos pesquisadores, das instituições proponente da pesquisa e das instituições financiadoras, para que se adote condutas direcionadoras e integradoras.

A pesquisa necessita de um aprendizado, geralmente longo e complexo. Em particular, são exigidas metodologias cada vez mais rigorosas, exigindo técnicas de análises cada vez mais sofisticadas. A produção do conhecimento, cada vez mais, se volta à interdisciplinaridade e ao multiprofissionalismo, deixando de ser uma ação solitária ${ }^{4}$.

A preocupação com o avanço das ciências reascende o debate dos desafios que o progresso científico exige. Dentre as várias abordagens possíveis dessa conjuntura da promoção do conhecimento, o contexto econômico e financeiro não pode ficar à 
margem, devido ao seu impacto nos direcionamentos da pesquisa. Esses interesses, principalmente os de origem privado, estão cada vez mais desejosos de que os subsídios investidos sejam recompensados com brevidade. É, portanto, uma abordagem de pesquisa que traz representantes de dois mundos muito diferentes para trabalhar $\mathrm{e}$ desenvolver relações profissionais estreitas ${ }^{5,6}$.

Assim, indagar sobre o elo que liga essas observações de normatização da ética na pesquisa, faz perceber, embora as normas regulamentadoras possam impor padrões para evitar conflitos de interesse e proteger os participantes de pesquisa, isso não mudará as pressões que os pesquisadores estão sujeitos. Segundo essa afirmação, se atribui à ética na pesquisa, uma tentativa de se estabelecer uma "ordem", unindo a sua dinâmica ao comprometimento do pesquisador.

\section{Ética e o contexto tecnocientífico}

A pesquisa, para ser ética não deve simplesmente obedecer às normas impostas. Os objetivos procurados e os meios usados para alcançá-la devem estar alinhados com as expectativas da pesquisa em sua relação com a sociedade. Esse alinhamento leva à reflexão sobre o que é aceitável e o que não é aceitável nas intervenções. Essa conduta diz respeito às responsabilidades do pesquisador com relação aos participantes da pesquisa. Este primeiro ponto afirma que a ética é, acima de tudo, uma tarefa crítica além de normativa ${ }^{7}$.

Um segundo ponto a ser observado, pretende salientar que negar o contexto tecnocientífico contemporâneo poderia reduzir a ética a uma utopia. Desse modo, as decisões não deveriam mirar no "ideal", e sim, serem negociadas dentro dos limites impostos pela responsabilidade ao coletivo. As violações desses limites implicam na perda de qualidade e potencializa a desconfiança dos resultados. Desse modo, poder-se-ia considerar a responsabilidade do pesquisador como um valor agregado ao produto da pesquisa ${ }^{8}$.

Os comitês de ética em pesquisa quando analisam os protocolos de pesquisa, assumem que o pesquisador se propõe a respeitar os princípios éticos da pesquisa, na expectativa de que as condutas propostas sejam suficientes para proteger os participantes recrutados e a própria lisura do processo de pesquisa proposto em seu conjunto. A resolução de outras questões éticas, especialmente aquelas que surgirão ao longo do projeto, será deixada a critério do pesquisador. Entende-se que isso não poderia ser diferente, uma vez que essas questões estão enraizadas no contexto da pesquisa e a resolução só pode ser fruto de uma reflexão que ocorre nesse contexto. Desse modo, questões éticas encontradas no caminho estão frequentemente relacionadas à orientação epistemológica assumidas pelo pesquisador ${ }^{9}$.

\section{A consolidação do sistema CEP/Conep}

No Brasil, a regulação da ética na pesquisa, desde o ano de 1996. é atribuição da Comissão Nacional de Ética em Pesquisa (Conep), criada pelo Conselho Nacional de Saúde (CNS). As Resoluções do CNS número $466 / 12^{10}$ e número $510 / 16^{11}$, aprovam as diretrizes regulamentadoras de pesquisas envolvendo seres humanos. A primeira, regulamenta pesquisas do modelo Biomédico e a segunda, regulamenta pesquisas de modelo de Ciências Humanas e Sociais.

A Conep, normatiza as pesquisas envolvendo seres humanos, organizando de modo sistêmico, um conjuntamente de rede descentralizada de comitês de ética (CEP), coordenados pelas instituições de abrigam pesquisas. Assim, o sistema se dedica em revisar as propostas de estudos com participantes humanos, objetivando garantir a conformidade dos estudos com as normas vigentes. A integração da Conep com os CEP foi denominada de sistema CEP/Conep ${ }^{10}$. O sistema CEP/Conep, em 2019, contava com 826 comitês de ética distribuídos em todo o território nacional.

No ano de 2018, o CEP Unioeste avaliou 790 protocolos de pesquisa, remanejados aos seus membros de acordo com a aproximação dos conteúdos das pesquisas com a área de atuação de cada membro. À época, somente nove CEP brasileiros avaliaram mais de um mil protocolos/ano e, somente dezessete 
avaliaram até 800 protocolos/ano ${ }^{12}$. Estima-se que, se nenhuma força contrária se manifestar, o CEP Unioeste, no biênio 2020/2021, deverá alcançar e/ou ultrapassar a análise de um mil pareceres/ano.

A atuação do CEP Unioeste no controle dos direitos exercidos pelas diversas partes interessadas na pesquisa, promove a verificação da conformidade das intervenções e do agir de acordo. Para tanto, algumas inconsistências de ordem burocrática e documental, que passaram a ser denominadas de "exigências cartorárias", necessitaram ser reguladas. Como resultado, o número de pendências na primeira submissão diminuiu substancialmente. A primeira submissão é entendida como a primeira avaliação de um
Relato de Experiência protocolo de pesquisa pelo colegiado do CEP. Mais recentemente, a análise preliminar mais rigorosa dos documentos na fase de aceitação dos documentos, provavelmente reforçou a tendência de diminuição do número de pendências na primeira submissão (Figura 1).

Essa análise documental preliminar não avalia as condutas de intervenções propostas e as suas adequações. Restringe-se unicamente às exigências "cartorárias". Realizada com duplo olhar, os documentos inadequados são retornados para saneamento. Após o saneamento, o protocolo segue para a análise dos pareceristas e, posteriormente, para a análise do Colegiado.

- Figura 1. Série histórica dos resultados dos pareceres do colegiado do CEP Unioeste, de 2015 a $2019 *$ (até o mês de setembro), em valores relativos, 2019.

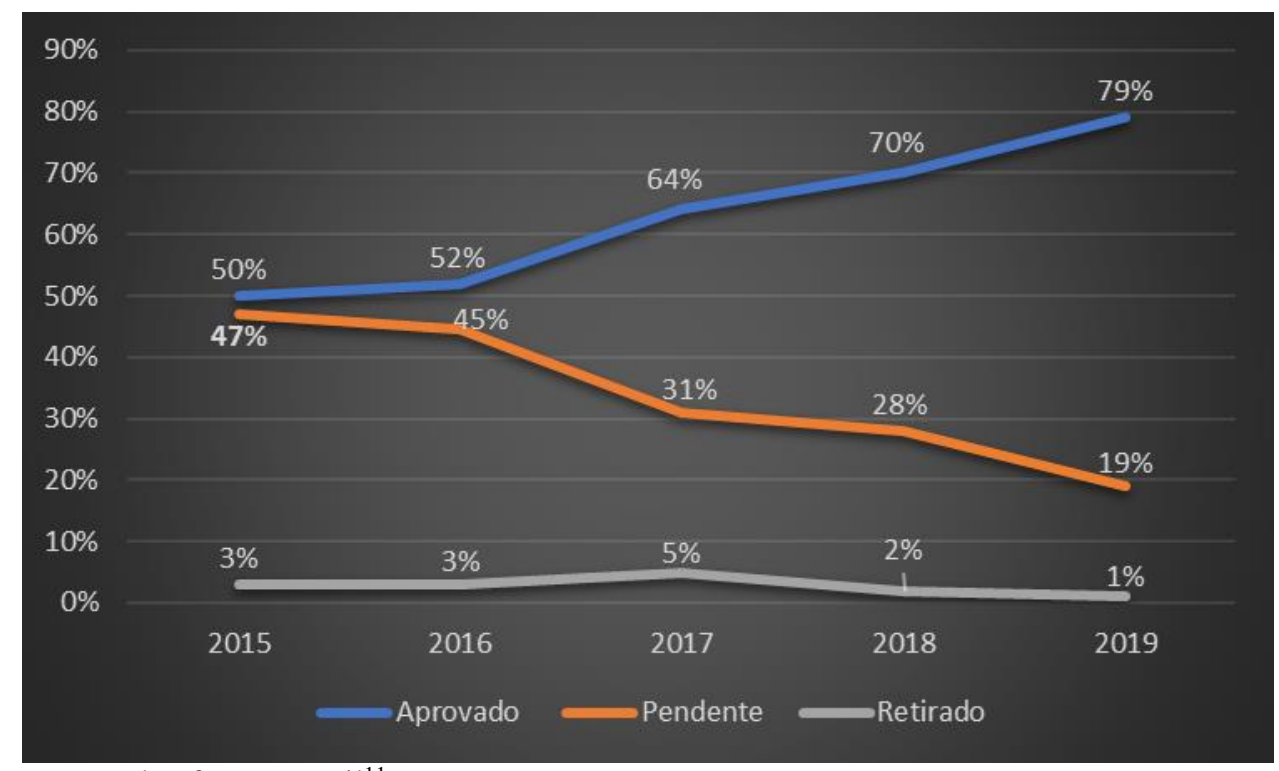

Fonte: Plataforma Brasil ${ }^{11}$.

O decréscimo de pendências observado reporta à questão: quais são, além das causas burocráticas (cartorárias), as causas que justificam a diminuição do menor número de pendências ética nas pesquisas avaliadas pelo CEP Unioeste? Muito embora a resposta para esta pergunta necessite de investigação específica, parece-nos plausível supor que o amadurecimento (aceitação) da figura do CEP na regulamentação das pesquisas e pelo próprio amadurecimento dos pesquisadores no trato com os procedimentos adotados pelo CEP, tenham contribuído para a ocorrência do fenômeno.
O CEP Unioeste procura a conciliação no entendimento conflitante entre os atores do processo, procurando a unanimidade colegiada nas tomadas de decisões. A votação de termo conflitante, para que a opinião da maioria vença, há tempo não faz parte da conduta colegiada. Desse modo, a colegialidade é baseada em uma postura de discussão e de entendimento que permite a redução da arbitrariedade, a presença de racionalidade das decisões, a transmissão de habilidades para além das pessoas e, finalmente, a desenvolvimento de conhecimento e reconhecimento mútuo. A 
ética pode se sustentar, em parte, estruturada em normas regulamentadoras, mas não pode ser reduzida a essa. $\mathrm{O}$ acionamento desse princípio de legalidade é insuficiente se não for complementada com o princípio da equidade, reconhecida como a segurança do julgamento na apreciação do que é devido proporcionalmente a cada um.

\section{CONCLUSÃO}

A problemática deste artigo concentrase na consolidação de elementos desafiadores ao comportamento de aspectos éticos na pesquisa. A nossa resposta se concentra na evolução do controle e no questionamento às diretrizes éticas que orientam a realização de pesquisas envolvendo seres humanos. Portanto, com base nas análises, colocamos o desempenho ético do CEP Unioeste ao atendimento prioritário de cinco elementos fundamentais: altruísmo, justiça, equidade, responsabilidade e colegialidade, que deve fundamentar a estrutura de consolidação do processo avaliativo e de seu progresso, sem, no entanto, aduzir $\mathrm{o}$ respeito às normas culturais do local.

Nosso padrão de conduta não pretende fazer com que os resultados éticos sejam um fim em si mesmo, tenta, pelo contrário, contornar barreiras, propondo um sistema de avaliação capaz de incentivar um processo de questionamento ético contínuo e sem exigências pétreas. Desse modo, a ação procedimental do CEP Unioeste pretende servir de estratégia para alavancar o compartilhamento de valores, sem instrumentalizar a avaliação dos aspectos éticos na pesquisa como fonte de heteronômica para o pesquisador. Da mesma forma, não oferecemos o único "modelo adequado" para impulsionar todo o desempenho ético de todo o sistema. Esse modelo, no melhor dos cenários, pode ser representativo do princípio de responsabilidade para melhorar o desempenho ético da pesquisa na instituição a qual pertencemos.

\section{REFERÊNCIAS}

Relato de Experiência

1. Malacarne V, Strieder DM, Lima DF. Ética, ciência e formação de professores: a escola na sociedade contemporânea. Rev. Ensaio. 2011 Set-Dez: 13(3): 51-66.

2. Organization for Economic Cooperation and Development - OECD. Research Ethics and New Forms of Data for Social and Economic Research. OECD Science, Technology and Industry Policy Papers. Paris: OECD Publishing; 2016.

3. Moore DA, Tenney ER. Time pressure, performance, and productivity. Research on Managing Groups and Teams, Berkeley. 2012; 15:305-326.

4. Scherer MDA, Pires DEP, Rémy J. A construção da interdisciplinaridade no trabalho da Equipe de Saúde da Família. Ciênc. saúde coletiva. 2013; 18(11):32033212.

5. Lima DF. A cumplicidade entre os comitês de ética em pesquisa em seres humanos e as ações investigatórias. Caderno de Educação Física. 2009; 8(14): 97-102.

6. Paraje, G. El financiamiento público de la investigación en salud en Chile. Revista médica de Chile. 2010: 138(1):36-43.

7. Campos, RHF. A pesquisa em ciências humanas, ciências sociais e educação: questões éticas suscitadas pela regulamentação brasileira. Educação e Pesquisa. 2020: 46: e217224.

8. Lima DF, Malacarne V. Ética em pesquisa envolvendo seres humanos: reflexões a partir das experiências da UNIOESTE - ciência e educação. ETD -Educação Temática Digital. 2009 Jul-Dez: 11 (2): 175-205.

9. Dal Rosso S, Bandeira I, Costa ATM. Pluralidade e Diversidade das Ciências Sociais: uma contribuição para a epistemologia da ciência. Soc. Estado. 2002; 17(2):231-246. 
10. Brasil. Ministério da Saúde. Conselho

Nacional de Saúde. Resolução no 466, de 12 de dezembro de 2012. Diário Oficial [da] República Federativa do Brasil, Brasília, DF, 12 dez. 2012, n. 12, 13 jun, p. 59. Seção 2, 2012.

11. Brasil. Ministério da Saúde. Conselho Nacional de Saúde. Resolução n ${ }^{\circ} 510$, de 7 de abril de 2016. Diário Oficial [da] República Federativa do Brasil, Brasília, DF, 24 maio 2016. Seção 1. p. 44-46, 2016.

12. Binsfeld PC. Desafios atuais do sistema CEP/CONEP. Formação Continuada dos Comitês de Ética em Pesquisa do RS, SC, PR, 2018 Set 4-6; Joinville, Brasil, Univille, 2018.

Recebido em: 04.05.2020. Aprovado em: 13.07.2020. 